\title{
A Temporal-Spatial Model-Based Method of Resilience Measurement for Mobile Ad Hoc Network
}

\author{
Weiqiang $\mathrm{Wu},{ }^{1}$ Ning Huang, ${ }^{1,2^{*}}$ Bo $\mathrm{Hu},{ }^{1}$ and Yue Zhang ${ }^{1}$ \\ ${ }^{1}$ School of Reliability and Systems Engineering, Beihang University \\ Beijing 100191, China \\ ${ }^{2}$ Science \& Technology on Reliability \& Environmental Engineering Laboratory \\ Beijing 100191, China
}

(Received May 2, 2016; accepted January 17, 2017)

Keywords: MANET, network fault tolerance, resilience measurement, temporal-spatial network model, routing protocols

Routing protocols are usually adopted as a kind of passive fault tolerance approach for a mobile ad hoc network (MANET) against failures of network links and nodes. General performancebased resilience measurement methods depend on whole network operation modeling, considering the network structure and routing protocols as packet delivery rules, thus leading to complex and inefficient network resilience measurements. Conversely, structure-based resilience measurement methods consider the removal of nodes and links as network failures in the network topology and have a higher efficiency than performance-based methods. Nevertheless, they ignore routing protocols as resilience impact factors, which result in a lower accuracy than performancebased methods. In this paper, we proposed a temporal-spatial network model-based method of resilience measurement, considering not only the network structure but also routing protocols as resilience impact factors. By analyzing the temporal-spatial characteristics of logical layers, which are formed with routes according to routing protocols, we built a temporal-spatial network model above the physical layer. Based on this model, resilience can be measured by the metric of temporal-spatial flow robustness (TSFR). Compared with the previous methods, our proposed method has a higher accuracy than the method based on physical flow robustness, which was originally considered the most accurate structure-based method, and it also has a higher efficient than the general performance-based measurement method.

\section{Introduction}

Mobile ad hoc networks (MANETs) are playing increasingly important roles in the areas of disaster relief, environmental sensing and monitoring, intelligent transportation, and military tactical systems. ${ }^{(1,2)}$ Mobility and wireless features of MANETs enable more use in a variety of areas; thus, high reliability and fault tolerance are required. Due to this demand, routing protocols have been assigned fault tolerance abilities ${ }^{(3,4)}$ according to rerouting, building multiroutes, and other approaches to handle failure nodes and links. Network resilience is considered to denote the tolerance ability of networks. ${ }^{(5,6)}$ Although a number of MANET routing protocols have been designed with fault tolerance features, they have not been considered resilience impact 
factors for resilience measurements. General network quantitative resilience measurement approaches, which include performance-based and structure-based methods, ${ }^{(5)}$ assess fluctuations in performance and topology as network failures. The performance-based methods consider performance metrics fluctuation, i.e., packet delivery ratio, which depends on whole network operation modeling, including packet generation and delivery. In addition, routing protocols are considered packet delivering rules without a resilience impact factor for performance-based methods. ${ }^{(6-8)}$ Consequently, they are highly complex while handling large-scale network resilience measurements and inefficient in supporting network designing and the development of routing protocols. To deal with these problems, ${ }^{(9)}$ over the past few years, a plethora of structure-based approaches and metrics have been proposed to measure network resilience. ${ }^{(5,9-11)}$ The common structure-based methods depend on the robustness evaluation of structural topology against attacks and failures. In the network science area, a series of metrics based on complex network robustness and corresponding methods have been proposed: Sydney et al. ${ }^{(12)}$ proposed a network resilience assessment method based on algebraic connectivity; Alenazi et al. ${ }^{(9)}$ introduced a method to evaluate network resilience based on spectral measure metrics, which is much more accurate than connectivity metrics for robustness assessment. ${ }^{(13)}$ Compared with methods based on performance metrics, the methods based on topology and structural metrics simplify packet generation and delivery while greatly reducing the complexity. Nevertheless, they are problematic in terms of accuracy because they ignore routing protocols as resilience impact factors.

A routing protocol is the primary way to guard against attacks and failures. In recent years, studies of structure-based resilience evaluation methods ${ }^{(14,15)}$ began to note the impact on the resilience of the routing protocol. It is noteworthy that Rohrer et al. ${ }^{(14)}$ found that networks with multipath routing protocols had a higher resilience than those with single-path routing protocols. However, they did not study the impact on resilience based on the discovery and maintenance mechanisms of routes for routing protocols, but they proposed a flow robustness metric that could be used to measure network resilience by considering the impact factor of routing protocols. This method also demonstrates a higher accuracy than previous structure-based metrics.

Overall, in the case of measurement methods for network resilience, the methods based on the performance are poor at measuring resilience for large-scale networks due to defects arising from complexity. The methods based on the network structure solve the complexity problem but show a low accuracy without considering routing protocols as the resilience impact factor. Considering protocols as an impact factor during the resilience measurement process is an effective method to enhance the accuracy of structure-based resilience evaluation methods.

Therefore, in this paper, we propose a novel method for measuring the resilience of MANETs, based on a temporal-spatial model and taking into consideration the routing protocol as a factor that impacts resilience. First, we constructed a network model for route discovery and maintenance mechanisms of routing protocols in a logic layer, which is above the physical layer, and then analyzed the resilience in terms of structural robustness metrics. Then, a contrast test was used to verify the accuracy and efficiency of the proposed model and method.

\section{Temporal-Spatial Network Model}

Based on the temporal-spatial features of routes running in a network, we proposed a temporal-spatial network model to model networks by considering routing protocols, which are the basis and object of resilience measurement approaches. 


\subsection{Temporal-spatial feature of the network}

Network transmission functions operate in routes according to routing protocols. The spatial feature of routes varies with time as shown in Fig. 1.

Thus, we can abstract the routes as a network according to routing protocols. Here, we considered physical connections exhibited on a physical layer. Based on physical connections, the logical connective is exhibited on a logical layer. For this model, dynamic features can be observed in temporal and spatial aspects; due to nodal features of movement, the physical links on a physical layer and the routes on a logical layer are not fixed. In addition, the fault tolerance mechanism against failures is another factor that can affect the spatial structure of networks. Therefore, the different routing protocols with different route discovery and maintenance mechanisms result in different structural behaviors of the abstracted networks of the routes; the structure spatial dynamic process is expressed in the time dimension.

Therefore, we considered the abstracted network formed by a routing protocol possessing temporal-spatial features and proposed a "temporal-spatial" network model. We use the temporal-spatial network model to describe variations in time and space according to routing protocols and the fault tolerance principle and carry out resilience measurements based on the network model as well.

\subsection{Definition of the temporal-spatial network model}

Route discovery and maintenance according to routing protocols depend on the connections in the physical layer, so the temporal-spatial network model also takes into account the physical network model. The connectivity of a MANET physical layer depends on the distance between nodes and the radius of signal transmission. There are several random graph models to model the physical layer of MANETs, such as Gilbert, Waxman, and Gabriel graphs. ${ }^{(9,16)}$ In this paper, we preferred using a Waxman graph for the model of the physical layer and our proposed temporalspatial network model for the logical layer.

Definition 1. Physical layer network model: The construction of the physical layer network should yield a Waxman model: ${ }^{(16)}$

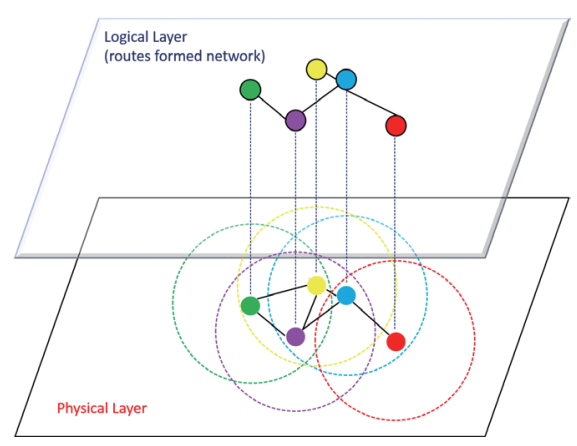

(a)

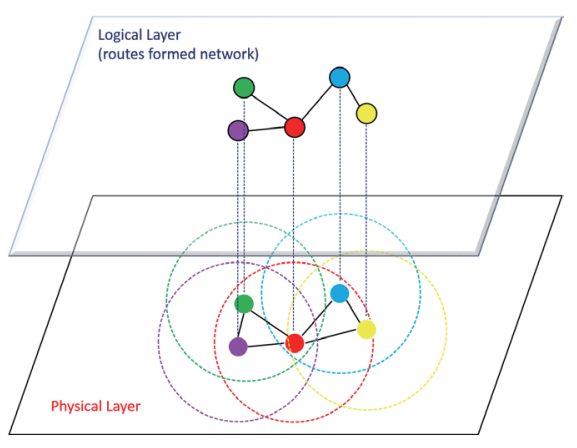

(b)

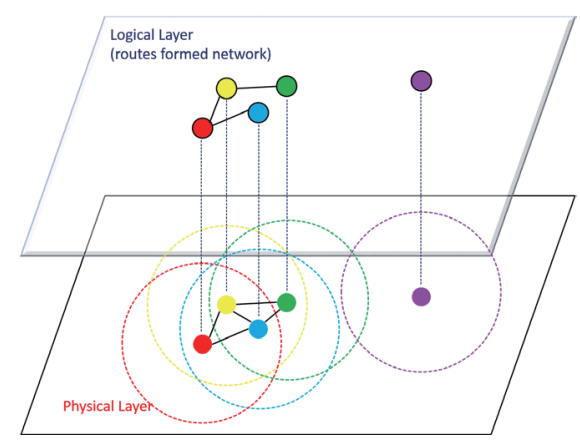

(c)

Fig. 1. (Color online) Different topologies of routes and links in different time profiles: (a) $t=t_{0}$, (b) $t=t_{1}$, and (c) $t=t_{2}$. 


$$
P(u, v)=\beta e^{\frac{-d(u, v)}{L \alpha}},
$$

where $\beta, \alpha \in(0,1]$ and $L$ is the maximum distance between any two nodes, $\beta$ denotes the density of links, and $\alpha$ corresponds to a high ratio of long links to short links.

Definition 2. Temporal-spatial network model: Here, we proposed the definition of the temporal-spatial network model: MANETs can be considered as a directed graph model, where $G$ $=(V, E) ; V$ is the set of nodes; $|V|$ is the number of nodes in the set; for $u \in V,\langle u, v\rangle$ denotes a valid path from $u$ to $v$, which is denoted by $e\langle u, v\rangle,(u, v)$ expressing the link between $u$ and $v$, denoted by $e(u, v) . E=\{e\langle u, v\rangle \mid u \in V, v \in V\}$ is defined as a set of valid edges. The number of edges or links is defined as $|E|$. The function $W: E \rightarrow R+, W(e\langle u, v\rangle)$ expresses the weight of the edge $e\langle u, v\rangle$, denoted by $w(u, v)$. If $w(u, v)$ is $+\infty$, the link $e\langle u, v\rangle$ is broken. $V:\left\{v_{1}, v_{2}, \ldots, v_{j-1}, v_{j}, v_{j+1}, \ldots, v_{n}\right\}$, denotes a path whose source node is $v_{1}$, destination node is $v_{n}$, and node number of the path is $n$. For $v_{i} \in v v_{j} \in$ $v, \operatorname{Path}\left(v_{i}, v_{j}\right)$ expresses the set of paths, whose source node is $v_{i}$ and destination node is $v_{j}$; in other words, these are the edge set of the temporal-spatial network. We assume $\operatorname{path}\left(v_{i}, v_{j}\right) \in \operatorname{Path}\left(v_{i}, v_{j}\right)$, and then define the function $f: \operatorname{Path}\left(v_{i}, v_{j}\right) \rightarrow R+$, where $f\left(\operatorname{path}\left(v_{i}, v_{j}\right)\right)$ denotes the evaluation function of $\operatorname{path}\left(v_{i}, v_{j}\right)$.

The purposes of the routing between source node $v_{i}$ and destination node $v_{j}$ are to find a $\operatorname{path}^{*}\left(v_{i}, v_{j}\right)$ for which the evaluation function is optimal and to satisfy the formula

$$
\exists \operatorname{path}^{*}\left(v_{i}, v_{j}\right) \forall \operatorname{path}\left(v_{i}, v_{j}\right), f\left(\operatorname{path}^{*}\left(v_{i}, v_{j}\right)\right) \leq f\left(\operatorname{path}\left(v_{i}, v_{j}\right)\right),
$$

subject to

$$
\operatorname{path}^{*}\left(v_{i}, v_{j}\right) \in \operatorname{Path}\left(v_{i}, v_{j}\right), \operatorname{path}\left(v_{i}, v_{j}\right) \in \operatorname{Path}\left(v_{i}, v_{j}\right) .
$$

Based on a network model, we can quantify the dynamic behavior of the temporal-spatial network structure by analyzing the following commonly used metrics:

Clustering coefficient: This is characterized by the degree of closeness of the nodes. A network with a high clustering coefficient shows a high efficiency. The formula is

$$
C_{i}=\frac{E_{i}}{\left(k_{i}\left(k_{i}-1\right)\right) / 2}=\frac{2 E_{i}}{k_{i}\left(k_{i}-1\right)} .
$$

To implement clustering coefficient analysis based on the temporal-spatial network model, we adopted the following steps. From the definition of the temporal-spatial model, we express the matrix $A[i, j]=\left(a_{i j}\right)_{M \times N}$. For the temporal-spatial network model considering routing protocols, we assume that $i, j$, and $k$ are the three vectors of the triangle included in network $A$. Then, $a_{i j} a_{j k} a_{k i}$ $=1$ or these three nodes cannot form a triangle, at which point $a_{i j} a_{j k} a_{k i}=0$. Hence, the number of triangles included in $A$ is

$$
E_{i}=\frac{1}{2} \sum_{j, k} a_{i j} a_{j k} a_{k i}=\sum_{k>j} a_{i j} a_{j k} a_{k i} .
$$

Based on the clustering coefficient formula, the clustering coefficient of node $i$ is 


$$
C_{i}=\frac{2 E_{i}}{k_{i}\left(k_{i}-1\right)}=\frac{1}{k_{i}\left(k_{i}-1\right)} \sum_{j, k=1}^{N} a_{i j} a_{j k} a_{i k}=\frac{\sum_{j \neq i, k \neq j, k \neq i} a_{j k} a_{i j} a_{i k}}{\sum_{j \neq i, k \neq j, k \neq i} a_{i j} a_{i k}},
$$

and the clustering coefficient of the entire network is

$$
C=\frac{1}{V} \sum_{i=1}^{N} C_{i}=\frac{\sum_{j \neq i, k \neq j, k \neq i} a_{j k} a_{i j} a_{i k}}{\sum_{j \neq i, k \neq j, k \neq i} a_{i j} a_{i k}}
$$

Ratio of largest component nodes: It is common to characterize the robustness of a network according to the network structure:

$$
R L C=\frac{\max \left\{C_{1}, C_{2}, \ldots, C_{n}\right\}}{N},
$$

where $C$ denotes the component of the network and $N$ denotes the total number of nodes in the network.

Since the network's structure and topology define the basic features of the network, based on the temporal-spatial network model, we can evaluate resilience by structure metrics considering the impact factor of the routing protocols.

\section{Method of Resilience Measurement}

From the perspective of network science, the structural features of a network can indicate a certain operational behavior. Therefore, if we consider resilience as part of the network's behavior, the network's structure will affect features of the resilience.

In addition to the metrics mentioned in Sect. 2.2, special metrics for evaluating the network's robustness and resilience taking into account the network structure metrics were also proposed, e.g., spectral metrics, nature connectivity, and algebraic connectivity. ${ }^{(6,13,17)}$ Although all of these are capable of measuring the robustness of the network structure, none completely reflects the impact of the routing mechanism on the network directly. Recently, scholars have begun to consider the impact of packet transmission and proposed flow robustness ${ }^{(14)}$ metrics to measure the flow robustness $(p h y F R)$ of the physical layer as an assessment of the resilience of a network, ${ }^{(10)}$ and compared flow robustness with other structure-based metrics. This method showed a higher accuracy than others:

$$
\text { phyFR }=\frac{\sum_{i=1}^{k} \mid \text { Component }_{i} \mid\left(\mid \text { Component }_{i} \mid-1\right)}{|n|(|n|-1)},
$$

where $G=(n, l)$ is the graph representing the given network and $\left\{\right.$ Component $\left._{i} ; 1<i<k\right\}$ is the set of components in graph $G$. The complexity for calculating the resilience using $p h y F R$ makes it difficult to find the size of the components in the network topology graph. Hence, we may count the number of edges instead of the number of nodes, because the numbers of links and edges are easy to collect during the simulation that considers the routing mechanism. 
Combining the metrics we introduced, the steps of our measurement method are as follows:

Step 1. Based on the temporal-spatial network model, build a spatial profile of the network, expressed as a matrix. The profile describes the connection status of the nodes in the temporalspatial network at each time step:

$$
A[i, j]= \begin{cases}w_{i j}, & \left\langle v_{i}, v_{j}\right\rangle \in E(G) \\ 0, & \left\langle v_{i}, v_{j}\right\rangle \notin E(G) .\end{cases}
$$

Step 2. Analyze the temporal-spatial flow robustness $(T S F R)$ network model.

$$
\operatorname{TSFR}=\frac{\sum_{i=1}^{k}\left|C_{i}\right|\left(\left|C_{i}\right|-1\right)}{|n|(|n|-1)}, 0 \leq \operatorname{TSFR} \leq 1,
$$

where the definitions of parameters follow those introduced in Eq. (9), but the object of analysis is the temporal-spatial network model. With the purpose of simplifying the complexity, we can also count edges or links instead of $C_{i}$. As we have known, the $n$ nodes can construct $n(n-1) / 2$ edges; hence, if $e_{i}$ is the edge number of $C_{i}$, Eq. (9) can be transformed to

$$
T S F R=\frac{\sum_{i=1}^{k} e_{i}}{E}
$$

where $E$ is the total number of possible network edges. Here, we proposed a simple proof:

$$
e_{i}=\frac{\left|C_{i}\right|\left(\left|C_{i}\right|-1\right)}{2}=\frac{\left|C_{i}\right|^{2}-\left|C_{i}\right|}{2} \Rightarrow\left|C_{i}\right|^{2}-\left|C_{i}\right|-2 e_{i}=0 .
$$

Solving Eq. (11),

$$
\begin{gathered}
\left|C_{i}\right|=\frac{1+\sqrt{1+8 e_{i}}}{2}, \\
\because \frac{|n|(|n|-1)}{2}=E, \\
\therefore|n|^{2}-|n|-2 E=0 \Rightarrow|n|=\frac{1+\sqrt{1+8 E}}{2} .
\end{gathered}
$$

By substituting Eqs. (14) and (16) into Eq. (11), we obtain Eq. (12).

Step 3. Measure the resilience of the network: Referring to the method of calculating resilience from Alenazi et al., ${ }^{(10)}$ we used the sum of the temporal-spatial flow robustness (STSFR) to denote the resilience: $\operatorname{STSFR}_{n}=\sum_{i=1}^{n} T S F R_{i}$. In comparison with the traditional structure-based measurement method, the sum of the physical layer flow robustness (SphyFR) metrics is introduced 
as a contrast: $\operatorname{Sphy} F R_{n}=\sum_{i=1}^{n} \operatorname{SphyFR_{i}}$.

\section{Case Study and Discussion}

We constructed a case study to verify the validity of the proposed model and a method to analyze $p h y F R$ and TSFR metrics by network resilience measurement.

\subsection{Introduction of the case and resilience assessment}

The case framework is based on a MANET for which an ad hoc on-demand distance vector (AODV) protocol is applied. With the purpose of trying to more closely approximate the real network usage profile and inspired by the simulation designed by Rohrer et al., ${ }^{(14)}$ we set up the simulation shown in Table 1.

Based on an AODV routing algorithm, the temporal-spatial network is generated and formed by valid routes and origin destination (OD) pair nodes as shown in Figs. 2 and 3.

Table 1

Simulation setup.

\begin{tabular}{lc}
\hline Attribute & Value \\
\hline Map size & $120 \times 120-180 \times 180 \mathrm{~m}^{2}$ \\
Node scale & 200 \\
Node communication radius & $64 \mathrm{~m}$ \\
Time steps & 200 \\
Movement mode & Random walk \\
Node failure mode & Random failure \\
Protocol & AODV \\
\hline
\end{tabular}

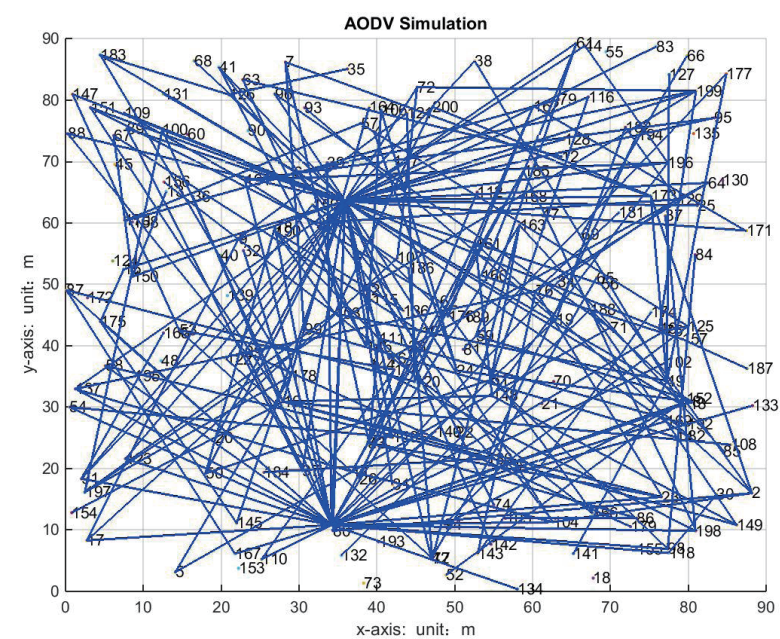

Fig. 2. (Color online) Available node number $=$ 200.

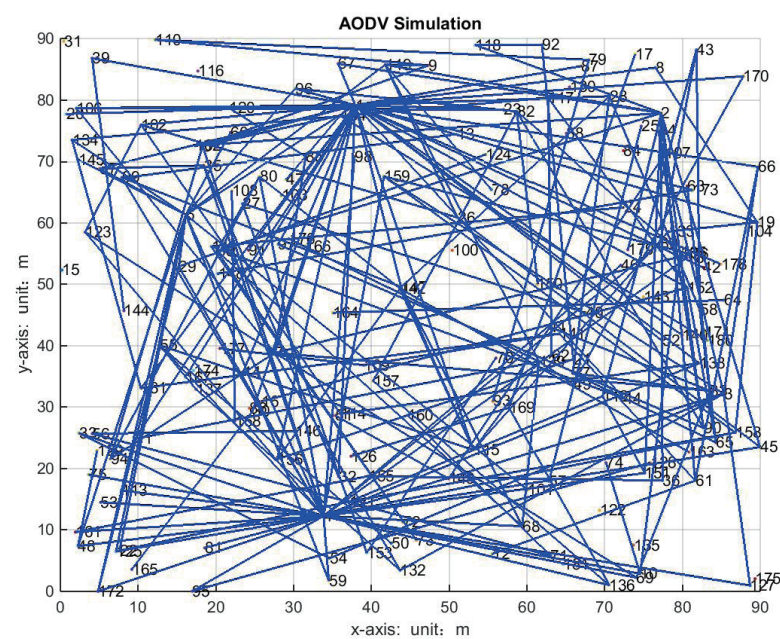

Fig. 3. (Color online) Available node number $=$ 180. 
After step 1 is completed, we can obtain the temporal-spatial network topology graph. By observing the topology graph at different time points, we note that the topology varies with increasing time, and the network structure metrics also change. Here, we express the temporalspatial network structure by the metrics discussed in Sect. 2.2 as shown in Fig. 4.

We collected the data for $p h y F R$, TSFR, SphyFR, and STSFR metrics during the simulation and show the results in Figs. 5 and 6. We selected six check points to show simulation results in Table 2.

From these results, we noted the following phenomenon:

1) Due to the structural features of the physical layer, which are indicated by different values of the $\alpha$ and $\beta$ parameters of the Waxman model of a physical layer model, the characteristics of robustness and resilience for a physical layer and a temporal-spatial abstract layer are different. To study the impact of the behaviors of different physical structures, we choose two groups of

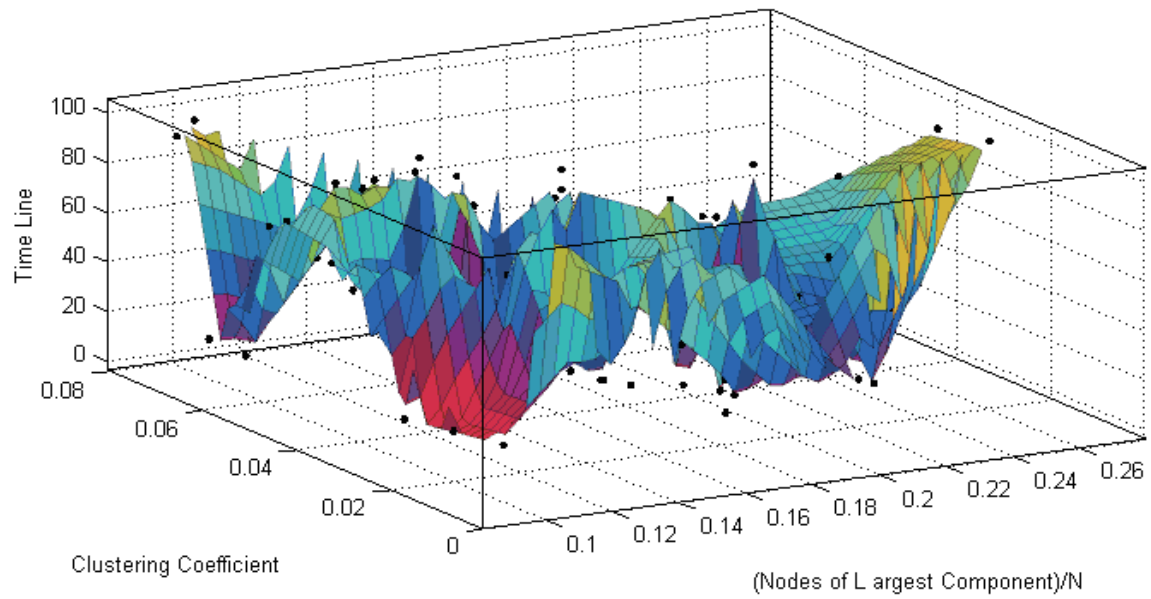

Fig. 4. (Color online) Clustering coefficient and largest component variation in the time dimension.
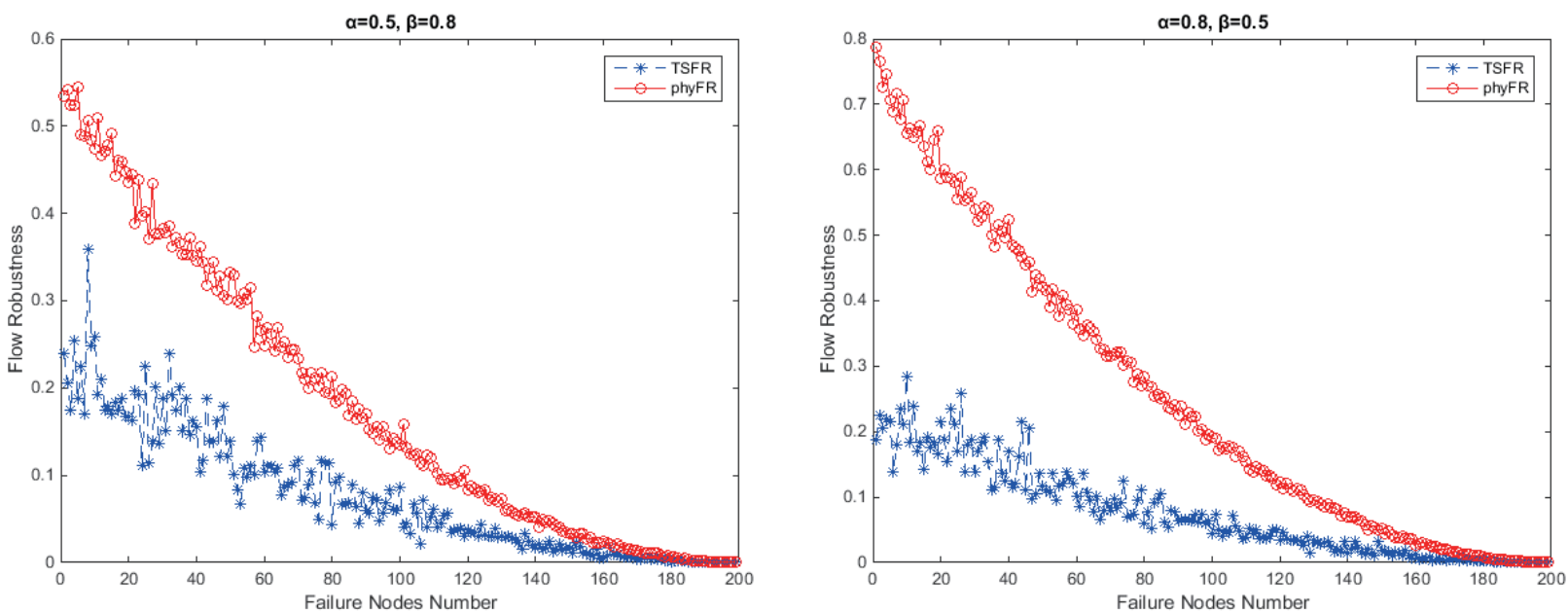

Fig. 5. (Color online) Comparison of simulated results for phyFR and TSFR. 

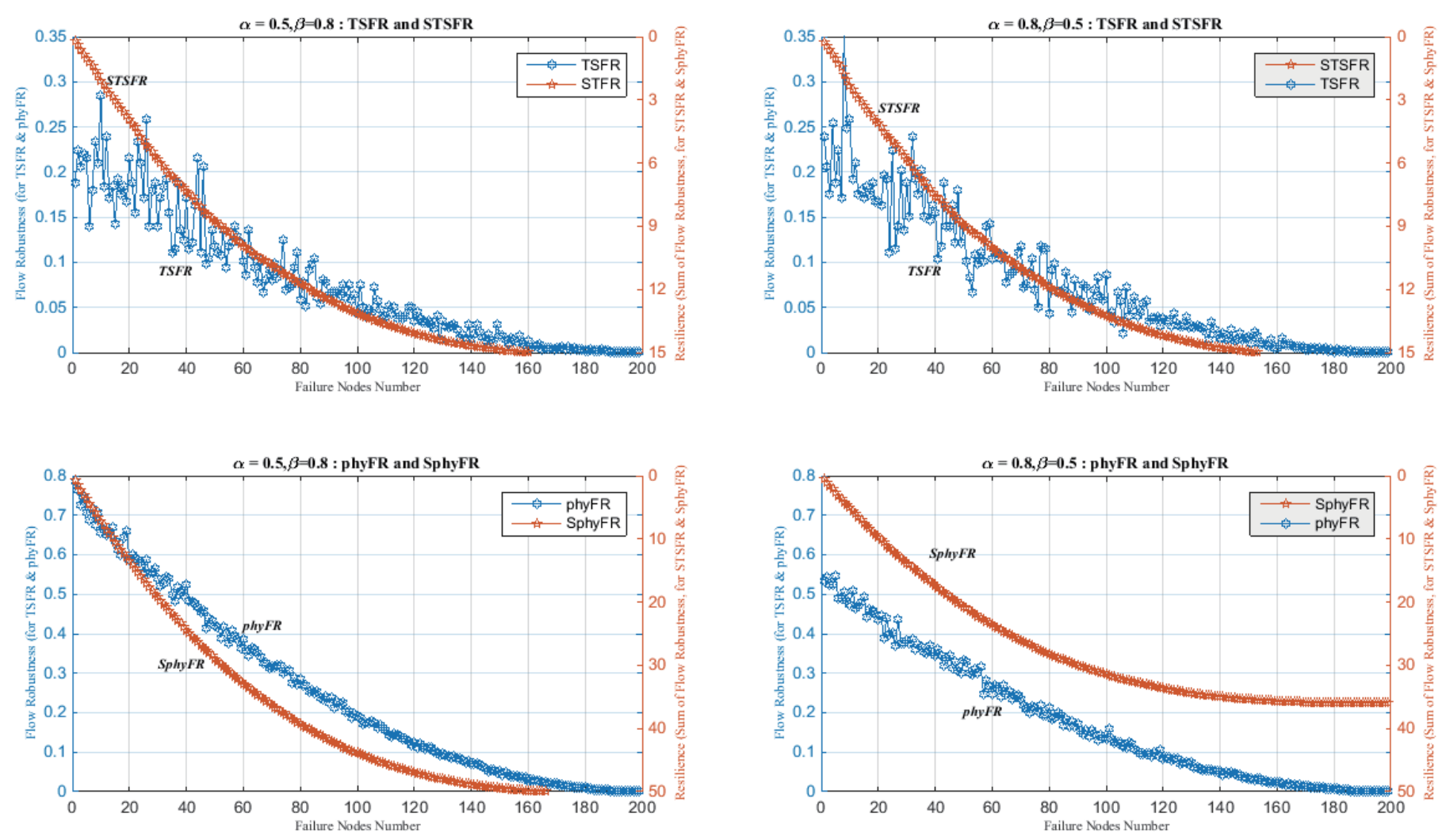

(a)

(b)

Fig. 6. (Color online) Resilience evaluation results under (a) $\alpha=0.5$ and $\beta=0.8$, and (b) $\alpha=0.8$ and $\beta=0.5$.

Table 2

Simulation results.

\begin{tabular}{|c|c|c|c|c|c|c|c|c|}
\hline \multirow{2}{*}{$\begin{array}{l}\text { Number of } \\
\text { failure nodes }\end{array}$} & \multicolumn{4}{|c|}{$\alpha=0.5, \beta=0.8$} & \multicolumn{4}{|c|}{$\alpha=0.8, \beta=0.5$} \\
\hline & TSFR & phyFR & STSFR & SphyFR & TSFR & phyFR & STSFR & SphyFR \\
\hline 1 & 0.188 & 0.787 & 0.188 & 0.787 & 0.239 & 0.535 & 0.239 & 0.535 \\
\hline 40 & 0.171 & 0.524 & 7.365 & 24.429 & 0.155 & 0.346 & 7.561 & 17.430 \\
\hline 80 & 0.059 & 0.284 & 11.732 & 39.297 & 0.043 & 0.214 & 11.888 & 28.189 \\
\hline 120 & 0.035 & 0.123 & 14.082 & 46.958 & 0.037 & 0.084 & 14.19 & 33.629 \\
\hline 160 & 0.012 & 0.027 & 14.998 & 49.831 & 0.005 & 0.025 & 15.060 & 35.664 \\
\hline 199 & 0 & 0 & 15.115 & 50.260 & 0 & 0 & 15.116 & 35.983 \\
\hline
\end{tabular}

$\alpha$ and $\beta$ values from Ref. $10: \alpha=0.5, \beta=0.8$ and $\alpha=0.8, \beta=0.5$. The network generally has higher robustness and resilience levels when $\alpha=0.5$ and $\beta=0.8$, and this result is consistent with the result in Ref. 10. Under the same physical layer setup, the values of TSFR and STSFR are lower than those of $p h y F R$ and $S p h y F R$, because the temporal-spatial abstract layer is above the physical layer, and an AODV routing protocol cannot use all physical connections but chooses some of them as routes.

2) When the number of failure nodes increases, the values of $p h y F R$ and $S p h y F R$ decrease more obviously than those of TSFR and STSFR, both of which consider the routing protocol factor. The reason for this effect is the fault tolerance ability of a routing protocol work against node failure. This reason may also explain why the impact of physical structures is much stronger for $p h y F R$ and SphyFR than for TSFR and STSFR (Table 2). 


\subsection{Validity verification and discussion}

Since the process of delivering packets takes the route into account, the TSFR metric should be much closer to the real robustness and resilience levels than the phyFR metric. To prove this assumption and verify the accuracy, we compared the simulated and experimental results of general performance-based approaches using the NS-3 platform. The configuration for the experiment is shown in Table 1. First, we set up the scenario on the NS-3 platform following Table 1. Once all source nodes finished sending the packets, we tracked and counted the number of successfully delivered packets from destination nodes.

To contrast the results and validate accuracy, we adopted a delivery ratio as a robustness metric for measuring the experimental results. The higher the packet delivery ratio, the stronger the fault tolerance ability. Therefore, we also adopted TSFR to compare the packet delivery ratio to validate accuracy. It is noteworthy that phyFR is the metric mentioned in Ref. 10 that is more accurate than other structural-based metrics for resilience evaluation. From Fig. 7, we observe that TSFR provides a closer assessment of the fault tolerance ability compared with the real ability (delivery ratio) than the general structure-based metric ( $p h y F R$ ). According to a Pearson correlation analysis, which is used to represent accuracy and was also adopted as an accuracy calculation method in Ref. 10, the accuracy of TSFR is 0.93 and that of phyFR is 0.83 . We also fitted the polynomial curve for both TSFR and phyFR from these fitting curves shown in Fig. 8. We observed that the robustness denoted by TSFR is closer than that denoted by phyFR. Hence, the resilience metric STSFR, which is based on TSFR, also has a higher accuracy than the general structural metrics.

Then, we verified the efficiency of the proposed method by comparing its source code complexity with that of the general performance-based method using NS-3. The source code of

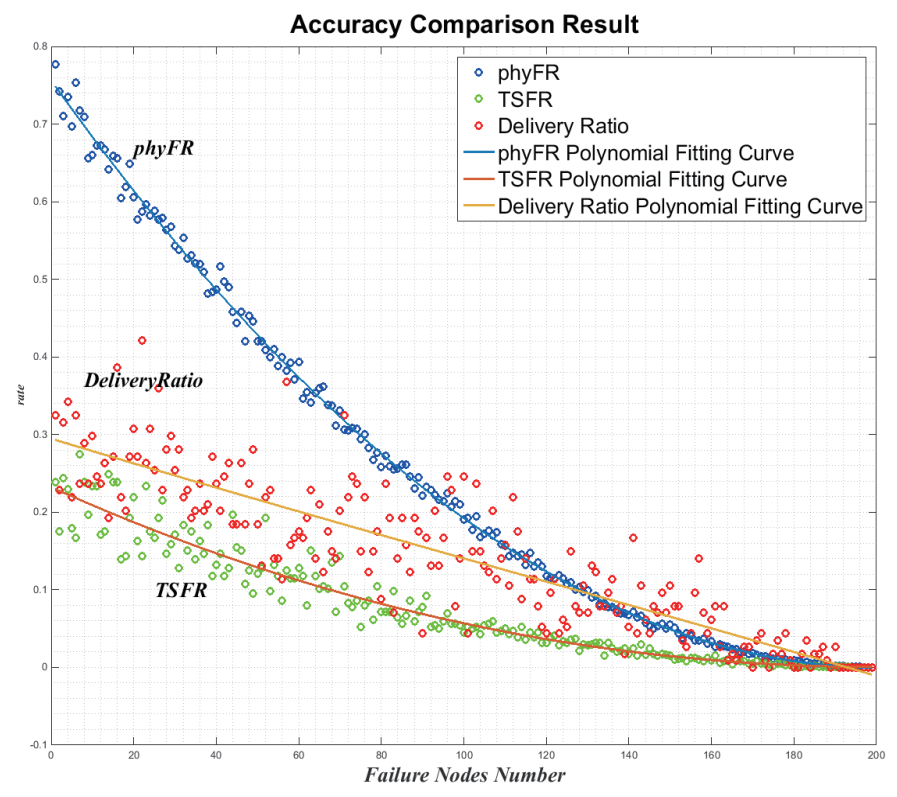

Fig. 7. (Color online) Accuracy validation using NS-3 for TSFR under the same scenario within 200 time steps. 

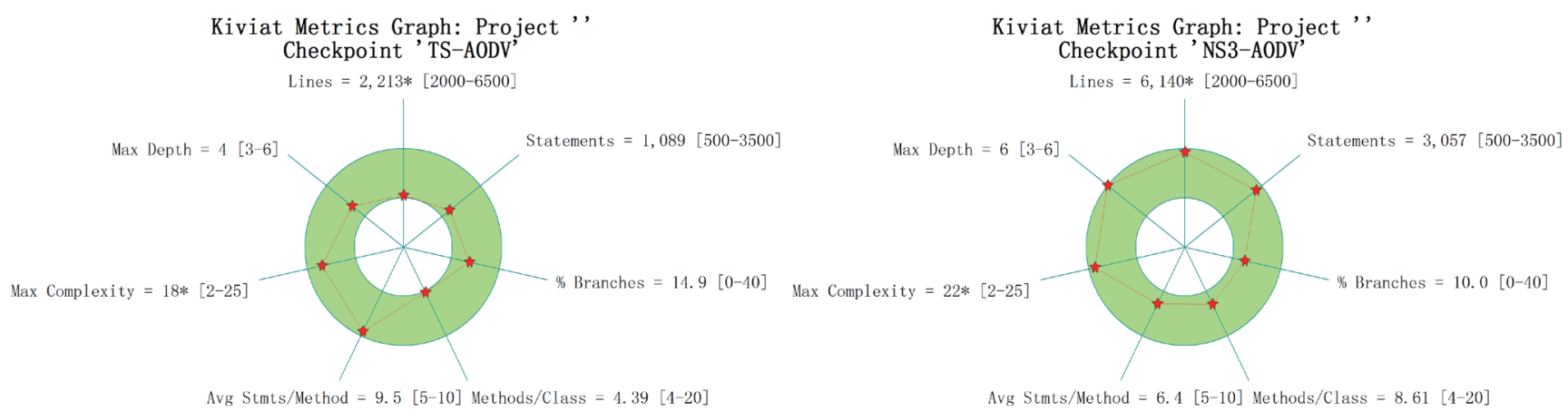

Fig. 8. (Color online) Complexity comparison with NS-3 source code for resilience measurement.

Table 3

Results of complexity analysis and comparison.

\begin{tabular}{lcc}
\hline Measured metrics & $\begin{array}{c}\text { Performance-based method } \\
\text { on NS-3 program }\end{array}$ & $\begin{array}{c}\text { Program of temporal-spatial } \\
\text { model-based method }\end{array}$ \\
\hline Line number & 6140 & 2213 \\
Percent branch & 14.9 & 10 \\
Methods per class & 4.39 & 8.61 \\
Average statement per method & 9.5 & 6.4 \\
Max complexity & 22 & 18 \\
Max depth & 6 & 4 \\
\hline
\end{tabular}

the temporal-spatial model-based method has the same features as that of the performance-based method. From the results of comparison (Table 3), our proposed method has a lower complexity and is expected to be more efficient than performance-based methods (Fig. 8).

\section{Conclusion}

In this paper, we propose a temporal-spatial model and its corresponding approach for MANET resilience measurement. Traditional structure-based measurement methods are limited in their accuracy because we do not know the factors from routing protocols' fault tolerance principles. To solve this problem, based on the construction of the model to describe routing protocols, we added a new upper layer above the physical layer, adopted from traditional structure-based resilience measurement methods, to reflect the impact of routing protocols on resilience. According to the contrast with general performance-based and structure-based resilience measurement methods, our measurement method shows a higher accuracy than the general structure-based methods and a higher efficiency than the general performance-based methods. In addition, according to case studies and results of simulation, the result of our proposed measurement method reflects the fault tolerance ability from routing protocols. It will be helpful to further study MANET resilience considering the combined impact of both physical and logic layers, and these results will be helpful for the design of networks and the development of routing protocols. 


\section{References}

1 S. Singh, S. C. Dutta, and D. K. Singh: Int. J. Res. Rev. Comput. Sci. (2012) 1654.

2 W. Wu, N. Huang, and Y. Zhang: Proc. 2015 Annu. Reliab. Maintainab. Symp. (RAMS) (IEEE, FL, 2015) pp. $1-6$.

3 K. Kaur and K. Kaur: Int. J. Comput. Appl. 120 (2015) 47.

4 P. Aggarwal: J. Global Res. Comput. Sci. 4 (2014) 42.

5 S. Hosseini, K. Barker, and J. E. Ramirez-Marquez: Reliab. Eng. Syst. Saf. 145 (2016) 47.

6 J. P. Sterbenz, D. Hutchison, E. K. Çetinkaya, A. Jabbar, J. P. Rohrer, M. Schöller, and P. Smith: Comput. Netw. 54 (2010) 1245.

7 J. P. Sterbenz, D. Hutchison, E. K. Çetinkaya, A. Jabbar, J. P. Rohrer, M. Schöller, and P. Smith: Telecommun. Syst. 56 (2014) 17.

8 D. Zhang and J. P. G. Sterbenz: Proc. 2015 7th Int. Workshop Reliab. Netw. Des. Model. (RNDM) (IEEE, 2015) p. 161.

9 M. J. F. Alenazi and J. P. G. Sterbenz: Proc. Resil. Week (RWS) 2015 (IEEE, 2015) p. 1.

10 M. J. F. Alenazi and J. P. G. Sterbenz: Proc. 11th Int. Conf. Des. Reliab. Commun. Netw. (DRCN 2015) (IEEE, NY, 2015) p. 157.

11 T. Dimitar, F. Sonja, M. Jani, and G. Aksenti: Proc. 12th IEEE Mediterr. Electrotech. Conf. (MELECON 2004) Vol. 2 (IEEE, 2004) p. 579.

12 A. Sydney, C. Scoglio, and D. Gruenbacher: Appl. Math. Comput. 219 (2013) 5465.

13 J. Wu, M. Barahona, Y. J. Tan, and H. Z. Deng: IEEE Trans. Syst. Man Cybern. Part A Syst. Humans 41 (2011) 1244.

14 J. P. Rohrer, A. Jabbarand, and J. P. G. Sterbenz: Telecommun. Syst. 56 (2013) 49.

15 M. Y. Su and C. W. Yang: Proc. 2015 Int. Conf. High Perform. Comput. Simul. (HPCS) (IEEE, 2015) p. 617.

16 B. M. Waxman: IEEE J. Sel. Areas Commun. 6 (1988) 1617.

17 J. P. Sterbenz, E. K. Çetinkaya, M. A. Hameed, A. Jabbar, S. Qian, J. P. Rohrer: Telecommun. Syst. 52 (2011) 705. 